\title{
Resonant Raman scattering in CdS(x)Se(1-x) nanocrystals: Effects of phonon confinement, composition and elastic strain
}

\begin{tabular}{|c|c|}
\hline Journal: & Journal of Raman Spectroscopy \\
\hline Manuscript ID: & JRS-10-0355 \\
\hline Wiley - Manuscript type: & Research Article \\
\hline $\begin{array}{r}\text { Date Submitted by the } \\
\text { Author: }\end{array}$ & 28-Sep-2010 \\
\hline Complete List of Authors: & $\begin{array}{l}\text { Freitas Neto, Ernesto; Universidade Federal de Uberlândia, Instituto } \\
\text { de Física } \\
\text { da Silva, Sebastião; Universidade de Brasília, Instituto de Física } \\
\text { Morais, Paulo; Universidade de Brasília, Instituto de Física } \\
\text { Vasilevskiy, Mikhail; Universidade do Minho, Departamento de } \\
\text { Fisica } \\
\text { Pereira-da-Silva, Marcelo; Universidade de São Paulo, Instituto de } \\
\text { Física de São Carlos } \\
\text { Dantas, Noelio; Universidade Federal de Uberlândia, Instituto de } \\
\text { Física }\end{array}$ \\
\hline Keywords: & $\begin{array}{l}\text { Resonant Raman scattering, } \mathrm{CdS}(\mathrm{x}) \mathrm{Se}(1-\mathrm{x}) \text { nanocrystals, } \\
\text { continuous lattice dynamics, atomic force microscopy, optical } \\
\text { absorption }\end{array}$ \\
\hline
\end{tabular}

\section{SCHOLARONE Manuscripts}




\title{
Resonant Raman scattering in $\mathrm{CdS}_{\mathrm{x}} \mathrm{Se}_{1-\mathrm{x}}$ nanocrystals: Effects of phonon confinement, composition and elastic strain
}

\author{
E. S. Freitas Neto ${ }^{*}$, S. W. da Silva ${ }^{2}$, P. C. Morais ${ }^{2}$, M. I. Vasilevskiy ${ }^{3}$, \\ M. A. Pereira-da-Silva ${ }^{4,5}$ and N. O. Dantas ${ }^{1}$ \\ ${ }^{1}$ Laboratório de Novos Materiais Isolantes e Semicondutores (LNMIS), Instituto de Física, \\ Universidade Federal de Uberlândia, CEP 38400-902, Uberlândia, MG, Brazil \\ ${ }^{2}$ Universidade de Brasília, Instituto de Física, Núcleo de Física Aplicada, CEP 70910-900, \\ Brasília, DF, Brazil \\ ${ }^{3}$ Centro de Física, Universidade do Minho, Campus de Gualtar, 4710-057, Braga, Portugal \\ ${ }^{4}$ Instituto de Física de São Carlos - USP, CEP 13560-250, São Carlos, SP, Brazil \\ ${ }^{5}$ Centro Universitário Central Paulista - UNICEP, CEP 13563-470, São Carlos, SP, Brazil \\ *Corresponding author: ernestosfn@yahoo.com.br (Ernesto S. Freitas Neto)
}

\begin{abstract}
Optical phonon modes confined in $\mathrm{CdS}_{\mathrm{x}} \mathrm{Se}_{1-\mathrm{x}}$ nanocrystal $(\mathrm{NC})$ quantum dots $(\approx 2 \mathrm{~nm}$ in radius) grown in a glass matrix by the melting-nucleation method, were studied by means of resonant Raman scattering (RRS) spectroscopy and theoretical modelling. The formation of nanocrystalline quantum dots (QDs) is evidenced by the observation of absorption peaks and theoretically expected resonance bands in the RRS excitation spectra. Since the underlying material is a ternary alloy, this system offers the possibility to investigate the interplay between the effects of phonon localization by disorder and phonon confinement by the NC/matrix interface. Based on the concept of propagating optical phonons, accepted for two-mode pseudo-binary alloys in their bulk form, we extended the continuous lattice dynamics model that has successfully been used for nearly-spherical NCs of binary materials, to the present case. After determining the alloy composition for NCs (that can be evaluated with only $2-3 \%$ uncertainty using the bulk longitudinal optical phonon frequencies) and the NC size (using atomic force microscopy and optical absorption data), the experimental RRS spectra were described rather well by this theory, including the lineshape and polarization dependence of the scattering intensity. Even though the presence of a compressive strain in the NCs, introduced by the matrix masks the expected downward shift owing to the phonons' spatial quantization, the asymmetric broadening of both Raman peaks is similar to that characteristic of NCs of pure binary materials. Although with some caution, we suggest that both CdSe-like and CdS-like optical phonon modes indeed are propagating within the NC size (and not localized by disorder at a shorter length scale) unless the alloy is considerably heterogeneous.
\end{abstract}

PACS: 73.21.La; 68.37.Ps; 71.35.Cc; 78.30.-j; 78.30.Er

Keywords: Resonant Raman scattering, $\mathrm{CdS}_{\mathrm{x}} \mathrm{Se}_{1-\mathrm{x}}$ nanocrystals; optical absorption, atomic force microscopy 


\section{Introduction}

Nanostructures based on II-VI semiconductors, presenting various morphologies, ranging from nanowires and nanobelts to nearly spherical quantum dots (QDs) are promising for nanophotonic devices operating in the visible spectral range. ${ }^{[1,2]}$ Pseudo-binary $\mathrm{CdS}_{\mathrm{x}} \mathrm{Se}_{1-\mathrm{x}}$ nanostructures are particularly attractive since their optical absorption and emission spectra can be tuned by taking advantage of both composition and size control in order to cover the entire visible spectral region, ${ }^{[3]}$ to optimize the magneto-optical properties ${ }^{[4]}$ or to introduce changes in the materials properties using ultra-fast laser pulses. ${ }^{[4]}$ Although colloidal chemistry techniques are now able to produce very high quality $\mathrm{CdS}$ and $\mathrm{CdSe}$ nanocrystals (NCs) possessing nearly spherical shape and discrete electronic spectra characteristic of QDs, ${ }^{[1]}$ this approach is not feasible for $\mathrm{CdS}_{\mathrm{x}} \mathrm{Se}_{1-\mathrm{x}}$ alloy NCs. In addition, there are demands for the synthesis of semiconductor nanoparticles buried in robust transparent matrices such as silica. ${ }^{[5]}$ The traditional melting-nucleation method emerges as a suitable approach as it allows for the fabrication of $\mathrm{CdS}_{\mathrm{x}} \mathrm{Se}_{1-\mathrm{x}} \mathrm{NCs}$ with controllable side and Se fraction inside borosilicate glass matrices. ${ }^{[6,7]}$

One important issue for the choice of $\mathrm{CdS}_{\mathrm{x}} \mathrm{Se}_{1-\mathrm{x}}$ nanostructures is the uniformity of the pseudo-binary alloy. Then, its electronic properties are simply intermediate between those of the binary end members. ${ }^{[8]}$ As known from the broad literature devoted to $\mathrm{Al}_{\mathrm{x}} \mathrm{Ga}_{1}$ ${ }_{x}$ As nanostructures, important effects like the surface segregation in pseudo-binary alloys can be probed by studying their phonon-related properties by means of far-infrared (FIR) and Raman spectroscopies. ${ }^{[9,10]}$ Raman studies of $\mathrm{CdS}_{\mathrm{x}} \mathrm{Se}_{1-\mathrm{x}} \mathrm{NCs}$ have been performed before, ${ }^{[7,11-15]}$ demonstrating the usefulness of Raman spectroscopy for the determination of the alloy composition in NCs. However, most of these studies were performed on relatively large nanoparticles ( $\mathrm{NC}$ radius $>3 \mathrm{~nm}$ ) where the contribution of optical phonons with significant non-zero wavenumbers is not readily seen in the Raman spectra. In this work, we study quite small NCs of two different compositions and the aim is to explore to what extent Raman-active phonon modes in alloy NCs can be described by the model considering (i) dispersive phonons in the bulk uniform pseudo-binary alloy and (ii) spatial quantization of these phonons in the small radius NCs where the mechanical confinement effect should be easier to observe. In other words, we wish to find out to what extent the lattice dynamics model, ${ }^{[16]}$ successfully applied to binary nanocrystal QDs ${ }^{[17,18]}$ is applicable to $\mathrm{CdS}_{\mathrm{x}} \mathrm{Se}_{1-\mathrm{x}} \mathrm{NCs}$. To the best of our knowledge, the above-mentioned approach has not yet been applied to alloy NCs where atomic substitution disorder is present. As a practical aspect of this work, we demonstrate that two independent parameters of alloy NCs, namely, composition and size, as well as their strain state can be determined by using a combination of data provided by optical absorption and Raman spectroscopies.

The paper is organized as follows. In the next section we present the essential information concerning sample preparation as well as the analysis of the optical absorption spectra. Section III is devoted to the Raman spectroscopy results. Section IV contains theoretical background, calculated Raman spectra and comparison with experiment. We conclude in Section V.

\section{NC fabrication and characterization}




\subsection{Sample preparation}

Samples were prepared using two different protocols hereafter called A and B. $\mathrm{CdS}_{\mathrm{x}} \mathrm{Se}_{1-\mathrm{x}}$ NCs were synthesized in the glass matrix SNAB with a nominal composition of $40 \mathrm{SiO}_{2}$ $.30 \mathrm{Na}_{2} \mathrm{CO}_{3} .1 \mathrm{Al}_{2} \mathrm{O}_{3} .29 \mathrm{~B}_{2} \mathrm{O}_{3}(\mathrm{~mol} \%)$ by adding either $2[\mathrm{CdO}+\mathrm{S}+\mathrm{Se}]($ wt $\%)$ or $8[\mathrm{CdS}+$ $\mathrm{CdSe}]($ wt \%) in the protocols $\mathrm{A}$ and $\mathrm{B}$, respectively. In both cases, the first step of the sample preparation consisted of melting the powder mixtures in an alumina crucible at $1200{ }^{\circ} \mathrm{C}$ for $30 \mathrm{~min}$. The crucible containing the melted mixture underwent quick cooling to room temperature. In the second step, a thermal annealing of the previously melted glass matrix was carried out at $560^{\circ} \mathrm{C}$ for several hours in order to enhance the diffusion of $\mathrm{Cd}^{2+}$, $\mathrm{S}^{2-}$ and $\mathrm{Se}^{2-}$ species into the hosting matrix , thus promoting the formation of $\mathrm{CdS}_{\mathrm{x}} \mathrm{Se}_{1-\mathrm{x}}$ NCs. The as-produced samples were labelled in two classes, A and B, according to the protocol used in the synthesis. They were characterized by atomic force microscopy (AFM), optical absorption, and then studied by resonant Raman scattering (RRS) spectroscopy.

\subsection{Atomic force microscopy}

Atomic force microscopy (AFM) images were obtained with a Multimode Nanoscope IIIa (Digital Instruments - Veeco). Figure 1 shows the AFM images with the corresponding NC size distributions, in three-dimensions (3D), for the samples A and B subjected to the longest thermal annealing, $20 \mathrm{~h}$ and $28 \mathrm{~h}$, respectively. The average radii estimated for $\mathrm{CdS}_{\mathrm{x}} \mathrm{Se}_{1-\mathrm{x}} \mathrm{NCs}$ from these AFM images were $R \approx 1.9 \mathrm{~nm}$ (sample $\mathrm{A}, 20 \mathrm{~h}$ annealing) and $R$ $\approx 2.2 \mathrm{~nm}$ (sample B, $28 \mathrm{~h}$ annealing) for the longest annealing time. The typical AFM morphology of isolated dots is shown in the detail of Fig. 1 as a two-dimensional (2D) picture, from which it is possible to observe the NCs' crystalline faces.

\subsection{Optical absorption spectra}

Room temperature optical absorption measurements were performed with a Varian-500 spectrometer at room temperature. Experimental absorption spectra of a whole set of samples A and B are presented in Fig. 2. The recorded spectra show a well-defined absorption peak associated with the lowest energy transition between quantum-confined electronic states in the $\mathrm{CdS}_{\mathrm{x}} \mathrm{Se}_{1-\mathrm{x}} \mathrm{NCs}$. The growth of $\mathrm{CdS}_{\mathrm{x}} \mathrm{Se}_{1-\mathrm{x}} \mathrm{NCs}$ in both classes of samples is clearly evidenced by the red shift of the absorption peak as the annealing time increases.

The absorption spectra, in combination with the Raman spectroscopy data presented in the next section, allow for an independent evaluation of the NC size, consistent with the AFM data. The required theoretical background is outlined below.

Within the effective mass approximation (EMA), the energy of the 8-fold degenerate lowest energy electron-hole (e-h) pair state (conventionally denoted $1 s_{e} 1 S_{3 / 2}$ ) in a quantum $\operatorname{dot}(\mathrm{QD})$ of radius $R$ is given by: ${ }^{[1]}$

$$
E_{1}(R)=E_{g}+\frac{\hbar^{2} \pi^{2}}{2 m_{e} R^{2}}+\frac{\hbar^{2} \chi_{1}^{2}}{2 m_{h h} R^{2}}-\zeta \frac{e^{2}}{\varepsilon_{0} R}+\Delta E_{\text {strain }}
$$


where $E_{g}$ is the bulk band gap energy, $m_{e}$ and $m_{h h}$ are the electron and heavy-hole effective masses, $\chi_{1}$ denotes the first root of a characteristic equation ${ }^{[19]}$ which depends on the light/heavy hole mass ratio, $\beta=m_{l h} / m_{h h}$, and varies between $\pi$ and 5.76. The term in Eq. (1) scaling as $R^{-1}$ represents the e-h correlation energy, with $\varepsilon_{0}$ and $\zeta$ denoting, respectively, the static dielectric constant of the QD material and a coefficient that varies between 1.77 and 1.91 depending on $\beta$. In our calculations we used the values of $\chi_{1}=5$ and $\zeta=1.8$.

The last term in Eq. (1) describes the effect of elastic strain eventually introduced by the surrounding matrix,

$$
\Delta E_{\text {strain }}=-3 K \frac{d E_{g}}{d P}\left(\frac{\delta a}{a}\right),
$$

where $K$ is the bulk modulus of the material, $\frac{d E_{g}}{d P}$ is the pressure coefficient of the band gap energy $\left(\approx 5 \times 10^{-6} \mathrm{eV} /\right.$ bar for both $\mathrm{CdS}$ and $\left.\mathrm{CdSe}^{[20]}\right), a$ is the lattice constant and $\delta a$ denotes its variation caused by the $\mathrm{NC}$ deformation. There are two factors originating the $\mathrm{NC}$ deformation, (i) NC-radius-dependent surface tension and (ii) NC-radius-independent deformation owing to different thermal expansion of the NC and matrix materials. According to Ref. [15], the combined result of these two effects can be described by the following type of relation,

$$
-\delta a / a=A\left(T_{g}-T\right)+B / R^{2}
$$

where $A$ and $B$ are constants and $T_{g}$ and $T$ are the sample growth and measurement temperatures, respectively. In principle, by virtue of Eq. (3) $\Delta E_{\text {strain }}$ depends on the QD radius.

The electronic properties of bulk $\mathrm{CdS}_{\mathrm{x}} \mathrm{Se}_{1-\mathrm{x}}$ are known to change continuously between those of the end member binary compounds. According to Refs. [21, 22], the band gap energy depends on composition in the following way:

$$
E_{g}(x)=x E_{g}^{(C d S)}+(1-x) E_{g}^{(C d S e)}-b_{1} x(1-x)-\Delta E_{2}(x),
$$

with $b_{1} \approx 220 \mathrm{meV}$ and $\Delta E_{2}(x) \leq 30 \mathrm{meV}$. As for the effective masses, no detailed data are available for intermediate compositions and we used the so called virtual crystal approximation (VCA) where the parameters vary just linearly between the corresponding values of CdSe and CdS. ${ }^{[8]}$ In our calculations we used $E_{g}$ obtained from Eq. (4) and the VCA values of $m_{e}$ and $m_{h h}$ (derived from the parameters given in Table I). The alloy composition was determined from the Raman spectra as explained below. By substituting the energy of the first peak in the measured absorption spectra (Fig. 2) into Eq. (1) and solving for $R$, while neglecting $\Delta E_{\text {strain }}$, we obtained the values of 1.95 and $2.18 \mathrm{~nm}$ for 
samples A (20 h annealing) and B (28 h annealing), respectively. However, if we take into account the strain which can also be estimated from the Raman spectra (Section IV), $\delta a / a \approx-0.01$, the estimated average radius is slightly higher, 2.05 and $2.29 \mathrm{~nm}$ for the samples A and B, respectively. These values agree quite well with the data obtained from the AFM images.

\section{Experimental RRS spectra}

Raman spectra were recorded at two temperatures (15 and $300 \mathrm{~K}$ ) using a JY-T64000 micro-Raman spectrometer in the backscattering geometry and employing several resonant lines of an $\mathrm{Ar}^{+}$laser. Figure 3 shows room temperature (RT) spectra of several samples of the $\mathrm{A}$ and $\mathrm{B}$ classes after performing different annealing time treatment. The formation of ternary $\mathrm{CdS}_{\mathrm{x}} \mathrm{Se}_{1-\mathrm{x}} \mathrm{NCs}$ in all cases is evidenced by the presence of the CdS-like and CdSelike LO-phonon-type Raman peaks. It can also be seen from Fig. 3 that the difference between the two peak frequencies $(\Delta \omega)$ remains approximately constant regardless of the thermal annealing time employed. This result is interesting since it indicates a good control over the growth kinetics of $\mathrm{CdS}_{\mathrm{x}} \mathrm{Se}_{1-\mathrm{x}} \mathrm{NCs}$, so that the composition ( $x$ ) remained nearly constant during the annealing procedure for both classes of samples (A and B). A similar behaviour has been reported in the literature only for $\mathrm{CdS}_{1-\mathrm{x}} \mathrm{Se}_{\mathrm{x}} \mathrm{NCs}$ with roughly equal content of substitutive $\mathrm{S}$ and $\mathrm{Se}$ chalcogen atoms $(0.4<\mathrm{x}<0.6){ }^{[14]}$

Figure 4 shows the dependence of the maximum scattering intensity upon the excitation wavelength, $\lambda_{I}$, and the corresponding absorption spectra are provided for comparison. The experimental points are fitted by theoretical resonance curves,

$$
F_{c}\left(\Omega_{I}, \omega\right)=C \int \frac{R^{-1}(E)}{\left(E-\hbar \Omega_{I}\right)^{2}\left(E-\hbar \Omega_{I}+\hbar \omega\right)^{2}} \exp \left[-\frac{\left(E-\bar{E}_{1}\right)^{2}}{2 \Delta_{E}^{2}}\right] \frac{d R}{d E} d E,
$$

where $C$ is a constant, $\Omega_{I}=2 \pi c / \lambda_{I}, \omega$ is the Raman shift (corresponding to one of the spectral peaks), $R(E)$ is the inverse of the function $E(R)$ given by Eq. (1), $\bar{E}_{1}$ is the photon energy corresponding to the absorption peak associated with the $1 s_{e} 1 S_{3 / 2}$ exciton transition, and $\Delta_{E}^{2}$ is the dispersion of the exciton ground state caused by the QD radius distribution. Equation (5) is a convolution of the resonant curve of a single QD with a Gaussian function describing inhomogeneous broadening because of the QD radius dispersion in the sample. (It will be derived in Section IV). Note that the maximum of the resonance curve is just slightly shifted with respect to the absorption peak and the experimental points follow Eq. (5) quite well. This provides strong evidence that the RRS process for both CdS-like and CdSe-like phonons indeed is mediated by the exciton states confined in the nanocrystal QDs.

Turning to the peak positions and spectral line shapes (see Fig. 5), we immediately notice that the characteristic phonon frequencies increase and the phonon damping decreases when the temperature is reduced, as expected. Several factors are to be taken into account for a more profound analysis of Raman-active phonon modes in NCs made of a ternary alloy semiconductor and embedded in a host matrix: $:^{[11,12]}$ 
(i) dependence of the bulk phonon spectra on the alloy composition,

(ii) quantum confinement effect on the phonon and exciton spectra, and

(iii) possible effects of elastic strain introduced in the NC by the surrounding matrix. In the next section we consider theoretical aspects of each of these factors.

\section{Theory and comparison with experiment}

\subsection{Bulk phonons}

We begin by describing the bulk optical phonons in $\mathrm{CdS}_{x} \mathrm{Se}_{1-x}$ alloys following the literature. ${ }^{[23]}$ According to the established classification, $\mathrm{CdS}_{\mathrm{x}} \mathrm{Se}_{1-\mathrm{x}}$ is a typical two-mode pseudo-binary alloy, that is, for any composition, $0<x<1$, there are two sub-bands of transverse (TO) and longitudinal (LO) optical phonons, called CdSe-like and CdS-like, respectively. The long-wavelength phonons belonging to these sub-bands are seen both in the FIR (TO modes) and Raman (LO modes) spectra. ${ }^{[23]}$ In the limit of $x \rightarrow 1$, the CdS-like sub-band resembles the optical phonon band of pure CdS whereas the CdSe-like one reduces to a local vibrational mode, i.e. its dispersion and the LO-TO splitting are vanishing. In the opposite limit of $x \rightarrow 0$ the two sub-bands change their roles.

It has been established that the long-wavelength TO phonon frequencies, $\omega_{T O}^{(j)}(x)$ (hereafter $j=1,2$ denotes the CdSe-like and CdS-like vibrations, respectively), vary approximately linearly between the corresponding limit values as $x$ changes between 0 and $1,{ }^{[24,25]}$

$$
\begin{aligned}
& \omega_{T O}^{(1)}(x)=\omega_{T O}^{(C d S e)}+\left[\omega_{l o c}^{C d S(S e)}-\omega_{T O}^{(C d S e)}\right] x \\
& \omega_{T O}^{(2)}(x)=\omega_{T O}^{(C d S)}+\left[\omega_{l o c}^{C d S e(S)}-\omega_{T O}^{(C d S)}\right](1-x) .
\end{aligned}
$$

The phonon-related dielectric function of a two-mode pseudo-binary semiconductor alloy usually is well described by the simple two-oscillator model, ${ }^{[9]}$

$$
\varepsilon(\omega, x)=\bar{\varepsilon}_{\infty}+\sum_{j=1,2} \frac{\rho_{j}(x) \omega_{T O}^{(j)}(x)^{2}}{\omega_{T O}^{(j)}(x)^{2}-\omega^{2}-i \omega \Gamma_{j}}
$$

where $\bar{\varepsilon}_{\infty}=(1-x) \varepsilon_{\infty}^{(1)}+x \varepsilon_{\infty}^{(2)}$ is the high-frequency dielectric constant, $\rho_{j}$ are oscillator strengths and $\Gamma_{j}$ are phenomenological damping parameters. It is usual to assume $\rho_{j}=\left(\varepsilon_{0}^{(j)}-\varepsilon_{\infty}^{(j)}\right) x_{j}$, where $\varepsilon_{0}^{(j)}$ is the static dielectric constant of the corresponding endmember material [for which the Lyddane-Sachs-Teller (LST) relation holds, $\left.\varepsilon_{0} / \varepsilon_{\infty}=\left(\omega_{L O} / \omega_{T O}\right)^{2}\right], x_{1}=1-x$ and $x_{2}=x$.

Using Eqs. (6) and (7), the corresponding bulk LO phonon frequencies of the alloy can be found as zeros of $\operatorname{Re} \varepsilon(\omega, x)$. The long-wavelength phonon frequencies, $\omega_{T O}^{(j)}(x)$ and $\omega_{L O}^{(j)}(x)$, calculated as described above and using the material parameters from Table I are presented on Fig. 6. Since the positions of the CdS-like and CdSe-like bulk-type modes, 
for a given alloy composition, can be affected by other factors such as strain, it was suggested $^{[24]}$ to use the difference between the CdS-like and CdSe-like LO phonon mode frequencies, $\Delta \omega_{L O}(x)=\omega_{L O}^{(2)}(x)-\omega_{L O}^{(1)}(x)$. This quantity is also plotted on Fig. 6 . Using the calculated $\Delta \omega_{L O}(x)$ dependence, we determined the approximate NC composition from the measured Raman spectra, $x_{A} \approx 0.6$ and $x_{B} \approx 0.2$ for the samples $\mathrm{A}$ and $\mathrm{B}$, respectively. These values will be defined more precisely after taking into account the phonon confinement effect.

Figure 6 also shows the frequencies of the electrostatic surface modes, defined by the equation,

$$
\varepsilon(\omega, x)=-\frac{l_{p}+1}{l_{p}} \varepsilon_{M}
$$

for $l_{p}=2\left(\varepsilon_{M}\right.$ is the dielectric constant of the surrounding medium). Modes with angular momentum number $l_{p}=2$ are supposed to be Raman-active in a spherical particle from the well-known symmetry arguments. ${ }^{[25]}$ Usually they were considered in the modelling of the Raman spectra of nearly spherical nanoparticles. ${ }^{[7,11-13]}$ Let us note that Eq. (8) takes into account only electrostatic confinement but not the mechanical boundary conditions. Therefore one should expect it to predict correct frequencies of the Raman-active quadripolar phonon modes only for rather large nanoparticles. We shall return to this point below.

\subsection{Phonon confinement}

As it is well known, the RRS of NCs consisting of a pure semiconductor material are determined by two factors, namely (i) the structure of spatially quantized electronic states that are resonantly excited by the incident light and (ii) the optical phonon modes interacting with these electronic states. If we assume that the $\mathrm{CdS}_{x} \mathrm{Se}_{1-x} \mathrm{NCs}_{\text {investigated in }}$ this study can be considered crystalline and approximately spherical in shape and the interaction between the e-h pairs and optical phonons described by the Fröhlich Hamiltonian, then phonon modes with angular momenta $l_{p}=0,2$ contribute to the scattering ${ }^{\dagger 1} .^{[18]}$ In general, these modes have a mixed longitudinal-transverse-interface nature, i.e. the displacement vector of such a mode contains each of these three components. They are classified, beyond the angular momentum, by two more "spherical" quantum numbers, $m_{p}$ and $n_{p}$. While the mode frequencies, hereafter denoted $\omega_{v}$, where $v=\left\{l_{p}, m_{p}, n_{p}\right\}$, are degenerate with respect to the momentum projection $m_{p}$ (unless anisotropy of the QD material is taken into account), they depend on the "radial" quantum number $n_{p}$. This dependence reflects the bulk optical phonon dispersion curves, namely,

\footnotetext{
${ }^{\dagger 1}$ Optical deformation potential (ODP) mechanism of the electron-phonon interaction gives rise to the participation of phonons with angular momenta 1 and 3 in the resonant Raman scattering. ${ }^{[17]}$ However, as shown in Ref. [26], the contribution of these modes can be neglected for most II-VI NCs because of the small value of the ODP characteristic constant.
} 
both the LO, $\omega_{L O}(\vec{q})$, and TO, $\omega_{T O}(\vec{q})$, phonon branches. The confined mode frequencies are distributed between $\min \left\{\omega_{L O}(\vec{q}), \omega_{T O}(\vec{q})\right\}$ and $\max \left\{\omega_{L O}(\vec{q}), \omega_{T O}(\vec{q})\right\}$ with the phonon wavevector $\vec{q}$ varying throughout the Brillouin zone. ${ }^{[17,18]}$ The phonon modes with $n_{p}>1$ are responsible for the asymmetric broadening of the Raman lineshape, characteristic of RRS in NCs.

If we wish to apply the same model, successfully used for semiconductor heterostructures $^{[16]}$ and first proposed for spherical QDs in Ref. [27], to NCs made of a pseudo-binary alloy, several questions arise. First, to what extent can one use the concept of phonon wavevector and bulk phonon dispersion curves in this case? Any random alloy is a disordered system and therefore, generally speaking, $\vec{q}$ is not a good quantum number. The literature reports on the discussion concerning this issue with respect to pseudo-binary semiconductor alloys, mostly in the case of $\mathrm{Al}_{x} \mathrm{Ga}_{1-x} \mathrm{As}$. The conclusion supported both experimentally $^{[28]}$ and by numerical calculations ${ }^{[29]}$ was that the band picture of the two bulk phonon modes (GaAs-like and AlAs-like, in the case of $\mathrm{Al}_{x} \mathrm{Ga}_{1-x} \mathrm{As}$ ) remains valid and well defined GaAs-like and AlAs-like phonon dispersions exist for any composition $x$. According to Ref. [30], Raman-active LO phonons in $\mathrm{Al}_{x} \mathrm{Ga}_{1-x} \mathrm{As}$ have well defined momenta and are coherent over distances of several tens of nanometres. Since $\mathrm{CdS}_{x} \mathrm{Se}_{1-x}$, similarly to $\mathrm{Al}_{x} \mathrm{Ga}_{1-x} \mathrm{As}$, is an exemplary two-mode alloy, we shall accept the validity of this conclusion in our case. It means that the spatial confinement effect on phonons in NCs of a few $\mathrm{nm}$ in size, consisting in the quantization of the phonon wavevector, is important and can be treated essentially in the same way as for NCs of pure binary materials. ${ }^{[18,27]}$ The second, practical question is what are the bulk phonon dispersion curves for the CdSe-like and CdS-like modes? Since such a data are not available, again, we shall use the analogy with the $\mathrm{Al}_{x} \mathrm{Ga}_{1-x} \mathrm{As}$ alloy. Regarding the alloying effect it has been shown ${ }^{[28,29]}$ that the phonon bands are shifted and the dispersion is smaller than the dispersion related to the end member compounds. Additionally, disorder also produces broadening of the Raman and FIR-active modes. Then, we shall assume that the band-edge LO and TO phonon frequencies shift as the composition $x$ changes in the same way as the corresponding Brillouin zone-centre frequencies. In other words, the bending parameters of all four bulk dispersion curves vary linearly with $x$ and vanish in the limit of $x \rightarrow 1$ for CdSe-like modes and $x \rightarrow 0$ for CdS-like ones. Given the relatively large difference between the frequencies of the CdSe-like and CdS-like modes, they can be considered independently.

According to the adopted model, phonon eigenmodes are determined by the coupled equations,

$$
\begin{aligned}
& \left(\omega^{2}-\omega_{T O}^{(j)^{2}}(x)\right) \vec{u}=\beta_{L O}^{(j)}(x) \vec{\nabla}(\vec{\nabla} \cdot \vec{u})-\beta_{T O}^{(j)}(x) \vec{\nabla} \times(\vec{\nabla} \times \vec{u})+\frac{\alpha^{(j)}(x)}{\bar{\rho}(x)} \vec{\nabla} \varphi ; \\
& \nabla^{2} \varphi=\frac{4 \pi \alpha^{(j)}(x)}{\bar{\varepsilon}_{\infty}(x)}(\vec{\nabla} \cdot \vec{u}) ; \quad j=1,2
\end{aligned}
$$

where $\vec{u}$ is the relative displacement of two sub-lattices, $\varphi$ denotes the electrostatic potential, $\beta_{T O}^{(j)}$ and $\beta_{L O}^{(j)}$ are phenomenological curvature parameters of the corresponding 
bulk phonon dispersion curves, defined as discussed above, $\bar{\rho}$ is the average reduced mass density of anion-cation pairs, and the polarisability of each mode is defined as

$$
\alpha^{(j)}(x)=\sqrt{\left.\bar{\varepsilon}_{\infty}(x) \bar{\rho}(x)\left(\omega_{L O}^{(j)^{2}}(x)-\omega_{T O}^{(j)}\right)^{2}(x)\right) /(4 \pi)} .
$$

As before, we used the standard electrostatic and rigid mechanical boundary conditions. Details of the calculation of the phonon eigenmodes can be found in Refs. [18, 31]. We have just generalized it to Eqs. (9) and (10). The designation of the eigenmodes, $v$, is now extended to include the branch number, $j$.

\subsection{Calculation of the Raman spectra}

Once the participating electron-hole states (the $1 s_{e} 1 S_{3 / 2}$ octet, in our case) and the Ramanactive phonon modes have been determined, the calculation of RRS spectra is straightforward. ${ }^{[17,18]}$ For the first-order Stokes process, the scattering probability per unit time and per incident photon is given by the following expression,

$$
W\left(\Omega_{I}, \omega\right)=\frac{(2 \pi)^{3}}{\Omega_{I} \Omega_{S} V^{2}}\left(\frac{e}{m_{0} \eta}\right)^{4} \sum_{v}\left[N\left(\omega_{v}\right)+1\right]\left|\sum_{k, k^{\prime}} \frac{\left(\vec{p}_{0 k} \cdot \vec{e}_{I}\right)\left(\vec{p}_{0 k^{\prime}}^{*} \cdot \vec{e}_{S}\right) B_{k k^{\prime}}^{v}}{\left(E_{k}-\hbar \Omega_{I}\right)\left(E_{k^{\prime}}-\hbar \Omega_{S}\right)}\right|^{2} \delta\left(\omega-\omega_{v}\right)
$$

In Eq. (11) $\Omega_{I}\left(\Omega_{S}\right)$ and $\vec{e}_{I}\left(\vec{e}_{S}\right)$ are the frequency and polarization vector of the incident (scattered) photon, respectively, $\omega=\Omega_{I}-\Omega_{S}$ is the Raman shift, $V$ is the QD volume, $m_{0}$ denotes the free electron mass, $\eta$ is the refraction index, $k$ enumerates different e-h pair states with the energy $E_{k}(R)$ and the momentum matrix element of the corresponding optical transition (denoted by $\left.\vec{p}_{0 k}\right)$, and $N\left(\omega_{v}\right)$ is the Bose-Einstein function. The matrix elements of the Fröhlich interaction, $B_{k k^{\prime}}^{v}$, proportional to $\alpha^{(j)}$, were calculated using the EMA wave functions with the VCA effective masses as explained in Ref. [18]. In the calculations the sum over $k$ was restricted to eight sub-states of the $1 s_{e} 1 S_{3 / 2}$ octet. As usual, the $\delta$-functions centred at the phonon frequencies were replaced by Lorentzians with a homogeneous width $\Gamma_{p h}$ (we took $\Gamma_{p h}=15 \mathrm{~cm}^{-1}$ ). The scattering probability determined by Eq. (11) was averaged over the QD radius distribution which was assumed Gaussian with the mean radius as determined from the absorption spectrum and the standard deviation of $10 \%$ (see below). The calculated spectra for two samples are shown in Figs. 7 and 8 together with the corresponding experimental ones. We shall compare them in the next section.

Let us derive the resonance function (5) for an ensemble of QDs with different radii. If we assume that the sub-states of the $1 s_{e} 1 S_{3 / 2}$ exciton state are degenerate, i.e. $E_{k}=E_{1}$ for all $k$, we get: 


$$
W\left(\Omega_{I}, \omega\right) \propto \frac{C_{F}^{2}}{\left(E_{1}-\hbar \Omega_{I}\right)^{2}\left(E_{1}-\hbar \Omega_{I}+\hbar \omega\right)^{2}},
$$

where $E_{1}$ is the ground state energy and $C_{F}$ is the exciton-phonon interaction constant, $C_{F} \propto R^{-1 / 2} \cdot{ }^{[18,27]}$ (Notice that $E_{1}$ and $C_{F}$ are the only parameters that depend on the QD radius.) Photon energies $\hbar \Omega_{I}=E_{1}$ and $\hbar \Omega_{S}=E_{1}$, the poles of Eq. (12), correspond to so called incoming and outgoing resonances. ${ }^{[10]}$ They can be resolved only if the inhomogeneous broadening is sufficiently small.

Let us assume a Gaussian distribution of QD radius,

$$
f_{R}(R)=\frac{1}{\sqrt{2 \pi \Delta_{R}^{2}}} \exp \left[-\frac{(R-\bar{R})^{2}}{2 \Delta_{R}^{2}}\right]
$$

where $\Delta_{R}^{2}$ is the radius dispersion. Then the RRS cross-section for the QD ensemble is:

$$
\frac{d^{2} \sigma}{d \omega d \Omega_{S}}\left(\Omega_{I}, \omega\right) \propto \int W\left(\Omega_{I}, \omega\right) f_{R}(R) d R .
$$

By substituting (12) and (13) and changing the integration variable in (14) we obtain Eq. (5) with $\Delta_{E}^{2}=(d E / d R)^{2} \Delta_{R}^{2}$, where $E(R)$ is given by Eq. (1).

\subsection{Discussion}

The expected effects of phonon confinement, seen in the calculated spectra, include the asymmetric broadening and the downward shift of the Raman peak positions with respect to the corresponding bulk LO phonon frequencies (see Table II). The calculated results qualitatively reproduce the main features of the experimental spectra, such as the width and the relative intensity of the two bands for two different compositions. It makes us confident that the general idea of our model is correct when assuming that there are dispersive optical phonons in a two-mode pseudo-binary alloy and that, consequently, the phonon-related properties of a QD made of such a material can be derived from the continuum lattice dynamics approach by applying appropriate spatial confinement conditions. We notice that the most important contribution to the polarized RRS spectra comes from sphericallysymmetric $\left(l_{p}=0\right)$ confined modes, as it has been demonstrated for QDs made of binary IIVI materials. ${ }^{[17,18]}$ The values of the downward shift of the Raman peak positions with respect to the corresponding bulk LO phonon frequencies are rather large in these smallradius NCs. It turns out that the difference between the frequencies of two Raman peaks in $\mathrm{CdS}_{x} \mathrm{Se}_{1-x} \mathrm{NCs}$ is only slightly affected by the phonon confinement effect. In fact, this difference, $\Delta \omega_{L O}=\omega_{L O}^{(C d S)}-\omega_{L O}^{(C d S e)}$, is only $2 \mathrm{~cm}^{-1}$ lower compared to the corresponding bulk alloy for sample A $(20 \mathrm{~h})$ and only $1 \mathrm{~cm}^{-1}$ higher for sample B $(28 \mathrm{~h})$. It means only a 2-3\% correction to the composition determined from "bulk" $\Delta \omega_{L O}(x)$ presented in Fig. 6. 
Concerning the quadripolar $\left(l_{p}=2\right)$ modes, they contribute significantly to the parallel-polarized spectra and are entirely responsible for the cross-polarization ones. These vibrations possess not only surface but also transverse and longitudinal components and have different frequencies classified according to different values of the radial quantum number, $n_{p}$. They do not coincide with those of the two electrostatic surface modes defined by Eq. (8). Only in the limit of large QD radius (large $n_{p}-\mathrm{s}$ ) and under the assumption that many e-h states contribute to the Raman scattering, the net effect of all $l_{p}=2$ vibrations can be described by the approximation of dispersion-less phonons leading to Eq. (8). (Similar situation takes place for FIR-active $l_{p}=1$ QD phonons as discussed in detail in Ref. [31].)

Parallel-polarized spectra have higher intensity than the crossed-polarized ones, as it is usual for spherical QDs ${ }^{[18]}$ and naturally explained by the fact that $l_{p}=0$ phonons contribute only to the former. The observed depolarization ratio $(0.3-0.4)$ is also typical for such systems and its precise value depends on several factors including QD size distribution and resonance conditions. The Raman band looks narrower in the parallelpolarized spectra because the frequencies of the (dominating) $l_{p}=0$ modes are limited to the LO branch of the bulk phonon dispersion curve, while the $l_{p}=2$ mode frequencies, in principle, occupy the whole range between $\omega_{T O}$ and $\omega_{L O}$. The asymmetry of the Raman bands, characteristic of all NC spectra and explained mainly by the contribution of phonon modes with $n_{p}>1^{[18]}$, is more clearly seen in the experimental spectra than in the calculated ones (Figs. 7 and 8). It could be due to significant deviations of the NC shape from spherical (i.e. shape disorder ${ }^{[18]}$ ), however, since the samples were grown at a rather high temperature, we think it is unlikely. It seems more plausible that the linear approximation assumed for the $x$ dependence of the Brillouin zone edge phonon frequencies used in the calculation of the Raman spectra is not quite correct and the dispersion of the CdS-like and CdSe-like phonon bands of bulk $\mathrm{CdS}_{\mathrm{x}} \mathrm{Se}_{1-\mathrm{x}}$ is larger than it follows from that approximation. Comparing the calculated and experimental Raman peak frequencies, there is a systematic shift between them (Table II). The values calculated for both samples are lower by approximately $10 \mathrm{~cm}^{-1}$ in all cases. Most likely this systematic shift is due to a compressive strain introduced in the NCs by the surrounding matrix. This effect has been previously reported for NCs embedded in borosilicate glasses ${ }^{[15,32,33]}$ and can be understood within the Grüneisen model ${ }^{[34]}$ of uniform relative shift of all vibration frequencies in a solid subjected to deformation,

$$
\delta \omega / \omega=-3 \gamma_{G r}(\delta a / a)
$$

where $\gamma_{G r}$ is the Grüneisen parameter. We see from Table II that the absolute value of the upward shift is somewhat higher for CdS-like modes (compared to the CdS-like ones), in agreement with Eq. (15). Since $\gamma_{G r} \approx 1.1^{[20]}$ and from Table II the upward shift of the stronger Raman peak is $\delta \omega_{\text {CdS-like }} \approx 12.7 \mathrm{~cm}^{-1}$ for the sample A and $\delta \omega_{\text {CdSe-like }} \approx 8.1 \mathrm{~cm}^{-1}$ for the sample B, we can evaluate a typical value of compressive strain in the NCs, 
$\delta a / a \approx-0.013$ and -0.012 for the samples $\mathrm{A}$ and $\mathrm{B}$, respectively. As mentioned in Section II, this strain results from two effects, namely, thermal expansion and surface tension. The latter should depend upon the QD radius [see Eq. (3)], even though we could not verify it quantitatively.

\section{Conclusion}

We measured and modelled resonant Raman spectra of nearly-spherical $\mathrm{CdS}_{x} \mathrm{Se}_{1-x} \mathrm{NCs}$ grown by the melting-nucleation method. The Raman-active phonon modes were derived from the continuum lattice dynamics approach by assuming that both CdSe-like and CdSlike optical phonon modes are propagating within the NC domain (unless either $x$ or $1-x$ is too small, in which case one of them reduces to a local mode). Since the excitation was performed in resonance with the absorption band associated with the lowest energy excitonic transition in the NCs, we used the EMA theory in order to explicitly describe the relevant electron and hole states in the QD. The measured RRS excitation spectra are in a very good agreement with the calculated resonance curves. The observed effects of temperature and scattering polarization on the spectra also are well understood theoretically. The general agreement between the theory and experiment in terms of line shapes and relative intensity of the $\mathrm{CdSe}$-like and $\mathrm{CdS}$-like modes is also rather good. However, the phonons' spatial quantization effect, which is a consequence of their (assumed) dispersive character in the bulk $\mathrm{CdS}_{x} \mathrm{Se}_{1-x}$ alloy, is masked by the presence of a further effect, namely, compressive strain in the NCs. It would be desirable to eliminate the strain effect in order to show unambiguously the propagating nature of the optical phonons in this two-mode pseudo-binary alloy as it was shown for $\mathrm{Al}_{x} \mathrm{Ga}_{1-x} \mathrm{As}$ using short-period superlattices. ${ }^{\left[{ }^{[2]}\right.}$ As a practical conclusion of this study, we note that the confinement and strain effects (opposite in sign of the shift that they produce) are similar for the CdSe-like and CdS-like modes. Therefore, one can evaluate the alloy composition for a NC with only a 2-3\% uncertainty from the NC Raman spectrum using the bulk $\Delta \omega_{L O}(x)$ curve. Therefore we propose the following recipe to evaluate the parameters of alloy NCs, namely, the composition and size, as well as their strain state by using a combination of optical absorption and Raman spectroscopies: (i) determine the alloy composition from the distance between the principal Raman peaks, (ii) given the composition, calculate the NC radius from the absorption peak position, (iii) estimate the NC strain from the position of the more intense Raman peak by comparing the experimental and theoretical values, and, if necessary, (iv) recalculate the NC radius from the absorption spectrum taking the strain effect into account. Finally, we would like to point out that the two variants of the meltingnucleation method of NC fabrication employed in this study revealed the good control the method provides over the alloy composition whereas the subsequent annealing procedure permits fine tuning of the NC size without affecting its composition.

\section{Acknowledgements}

The authors acknowledge the financial support from FAPEMIG and MCT/CNPq (Brazil), and the Portuguese Foundation for Science and Technology (FCT). 


\section{References}

[1] A. L. Rogach (ed.), Semiconductor Nanocrystal Quantum Dots. Synthesis, Assembly, Spectroscopy and Applications, Springer, New York, 2008.

[2] Y. L. Kim, J. H. Jung, K. H. Kim, H. S. Yoon, M. S. Song, S. H. Bae, Y. Kim, Nanotechnology 2009, 20, 095605.

[3] D. Magana, X. Wei, G. F. Strouse, Phys. Rev. B 2008, 77, 115337.

[4] R. Osellame, R. Martinez Vazquez, G. Cerullo, R. Ramponi, O. Svelto, V. Russo, A. Li Bassi, C. E. Bottani, C. Spinella, Phys. Rev. B 2007, 76, 045340.

[5] P. Huber, H. Karl, B. Stritzker, Appl. Surf. Sci. 2006, 252, 2497.

[6] M. H. Yükselici, J. Phys.: Condens. Matter 2001, 13, 6123.

[7] A. M. Alcalde, A. A. Ribeiro, N. O. Dantas, D. R. Mendes Jr, G. E. Marques, C. Trallero-Giner, Brazilian Journal of Physics 2006, 36, 832.

[8] O. Madelung (ed), Semiconductors: Properties of II-VI Compounds, Springer, Berlin, 1982.

[9] T. Dumelow, T. J. Parker, S. R. P. Smith, D. R. Tilley, Surf. Sci. Rep. 1993, 17, 151.

[10] M. Cardona, In: Light Scattering in Solids II, edited by M. Cardona and G. Güntherodt, Topics in Applied Physics, Vol. 50, Chap. 2, Springer-Verlag, Berlin, 1982.

[11] A. Roy, A. K. Sood, Phys. Rev. B 1996, 53, 12127.

[12] A. V. Gomonnai, Yu. M. Azhnyuk, V. O. Yukhymchuk, M. Kranjcec, V. V. Lopushansky, Phys. Stat. Sol. (b) 2003, 239, 490.

[13] A. Mlyah, A. M. Brugman, R. Carles, J. B. Renucci, M. Ya. Valakh, A. V. Pogorelov, Sol. Stat. Comm. 1994, 90, 567.

[14] Yu. M. Azhnyuk, A. V. Gomonnai, Yu. I. Hutych, V. V. Lopushansky, I. I. Turok, V. O. Yukhymchuk, D. R. T. Zahn, J. Crystal Growth 2010, 312, 1709.

[15] G. Scamarcio, M. Lugara, D. Manno, Phys. Rev. B 1992, 45, 13792.

[16] C. Trallero-Giner, R. Pérez-Alvarez, F. García-Moliner, Long Wave Polar Modes in Semiconductor Heterostructures, Pergamon, London, 1998.

[17] A. G. Rolo, M. I. Vasilevskiy, M. Hamma, C. Trallero-Giner, Phys. Rev. B 2008, $78,081304$. 
[18] A. G. Rolo, M. I. Vasilevskiy, J. Raman Spectroscopy 2007, 38, 618.

[19] Al. L. Efros, M. Rosen, M. Kuno, M. Nirmal, D. J. Norris, M. G. Bawendi, Phys. Rev. B 1996, 54, 4843.

[20] Landolt-Börnstein Numerical Data and Functional Relationships in Science and Technology, Group III, Vol. 17, Springer-Verlag, Berlin, 1982.

[21] S. Permogorov, A. Reznitsky, J. Luminescence 1992, 52, 201.

[22] A. Klochikhin, A. Reznitsky, S. Permogorov, T. Breitkopf, M. Grün, M. Hetterich, C. Klingshirn, V. Lyssenko, W. Langbein, J. M. Hvam, Phys. Rev. B 1999, 59, 12947.

[23] I. F. Chang, S. S. Mitra, Phys. Rev. 1968, 172, 924.

[24] A. Tu, P. D. Persans, Appl. Phys. Lett. 1991, 58, 1506.

[25] E. Duval, Phys. Rev. B 1992, 46, 5795.

[26] M. I. Vasilevskiy, C. Trallero-Giner, Phys. Stat. Sol. B 2010, 247, 1488.

[27] E. Roca, C. Trallero-Giner, M. Cardona, Phys. Rev. B 1994, 49, 13704.

[28] B. Jusserand, D. Paquet, F. Mollot, Phys. Rev. Lett. 1989, 63, 2397.

[29] S. Baroni, S. de Gironcoli, P. Giannozzi, Phys. Rev. Lett. 1990, 65, 84.

[30] J. A. Kash, J. M. Hvam, J. C. Tsang, T. F. Kuech, Phys. Rev. B 1988, 38, 5776.

[31] M. I. Vasilevskiy, Phys. Rev. B 2002, 66, 195326.

[32] Y. N. Hwang, S. Shin, H. L. Park, S. H. Park, U. Kim, H. S. Jeong, E. J. Shin, D. Kim, Phys. Rev. B 1996, 54, 15120.

[33] E. S. F. Neto, N. O. Dantas, S. W. da Silva, P. C. Morais, M. A. Pereira-da-Silva, J. Raman Spectrosc. 2010, DOI: 10.1002/jrs.2565.

[34] M. Born, K. Huang, Dynamical Theory of Crystal Lattices, Clarendon, Oxford, 1996. 
Table I. Material parameters $(T=300 \mathrm{~K})^{\dagger 2}$

\begin{tabular}{|c|c|c|c|c|c|c|c|c|}
\hline $\begin{array}{l}\text { Para- } \\
\text { meter }\end{array}$ & $E_{g}, \mathrm{eV}$ & $m_{e} / m_{0}$ & $m_{h h} / m_{0}$ & $\begin{array}{c}\omega_{T O}, \\
\mathrm{~cm}^{-1}\end{array}$ & $\begin{array}{c}\omega_{L O}, \\
\mathrm{~cm}^{-1}\end{array}$ & $\begin{array}{c}\omega_{l o c}, \\
\mathrm{~cm}^{-1}\end{array}$ & $\varepsilon_{\infty}$ & $\begin{array}{c}K, 10^{10} \\
\mathrm{~Pa}\end{array}$ \\
\hline $\mathrm{CdSe}$ & 1.75 & 0.13 & 0.45 & 168 & 210 & $(\mathrm{~S}) 266$ & 6.10 & 5.50 \\
\hline $\mathrm{CdS}$ & 2.50 & 0.20 & 0.70 & 238 & 302 & $\begin{array}{c}(\mathrm{Se}) \\
185\end{array}$ & 5.32 & 6.15 \\
\hline
\end{tabular}

Table II. Calculated and experimental Raman peak frequencies $\left[\mathrm{cm}^{-1}\right]$

\begin{tabular}{|l|c|c|c|c|c|c|c|}
\hline Sample & $\begin{array}{c}R, \\
\mathrm{~nm}\end{array}$ & $\begin{array}{c}\text { CdSe-like } \\
\text { bulk }\end{array}$ & $\begin{array}{c}\text { CdSe-like } \\
\text { NC }\end{array}$ & Experim. & $\begin{array}{c}\text { CdS-like } \\
\text { bulk }\end{array}$ & $\begin{array}{c}\text { CdS-like } \\
\text { NC }\end{array}$ & Experim. \\
\hline $\mathrm{A}(x \approx 0.6)$ & 1.7 & 190.0 & 185.3 & 195.6 & 295.5 & 288.7 & 301.4 \\
\hline $\mathrm{B}(x \approx 0.2)$ & 2.1 & 200.4 & 196.3 & 204.4 & 279.7 & 276.7 & 286.8 \\
\hline
\end{tabular}




\section{Figures captions}

Figure 1. (colour online) AFM images and size distributions of $\mathrm{CdS}_{\mathrm{x}} \mathrm{Se}_{1-\mathrm{x}} \mathrm{NCs}$ embedded in a sample A (a) and a sample B (b), subjected to thermal annealing at $560^{\circ} \mathrm{C}$ for $20 \mathrm{~h}$ and $28 \mathrm{~h}$, respectively. A 2D image of isolated NCs is shown in detail.

Figure 2. (colour online) Room temperature absorption spectra of samples A (a) and B (b) subjected to thermal annealing at $560^{\circ} \mathrm{C}$ for several hours. For comparison, it is also shown the absorption spectrum of SNAB glass matrix (bottom).

Figure 3. (colour online) Unpolarized RRS spectra recorded at room temperature from samples A (a) and samples B (b) subjected to thermal annealing at $560^{\circ} \mathrm{C}$ for different time as indicated.

Figure 4. (colour online) Absorption spectra of the samples (a) A and (b) B subjected to thermal annealing for 20h. Resonant Raman curve of the corresponding samples (c) A and (d) B obtained from several excitation wavelengths.

Figure 5. (colour online) Experimental RRS spectra $\left(\lambda_{I}=488 \mathrm{~nm}\right.$ ) with (a) parallel and (b) cross polarisations recorded at two different temperatures from the sample B subjected to thermal annealing for $20 \mathrm{~h}$.

Figure 6. (colour online) Calculated frequencies of bulk optical phonons and electrostatic surface modes for $\mathrm{CdS}_{\mathrm{x}} \mathrm{Se}_{1-\mathrm{x}}$ alloys of different composition. Also shown is the difference between the frequencies of the CdS-like and CdSe-like LO modes. The surface mode frequencies were calculated assuming $\varepsilon_{M}=2.25$ for the surrounding medium.

Figure 7. (colour online) Experimental ( $\lambda_{I}=488 \mathrm{~nm}$, panel a) and calculated (b) RRS spectra of sample A (annealed for $20 \mathrm{~h}$ ) for two polarizations of the scattered light. The inset shows relative contributions of the different confined phonon modes with angular momenta 0 and 2 to the parallel polarisation spectrum.

Figure 8. (colour online) Same as in Fig. 7 for sample B annealed for $20 \mathrm{~h}$. 


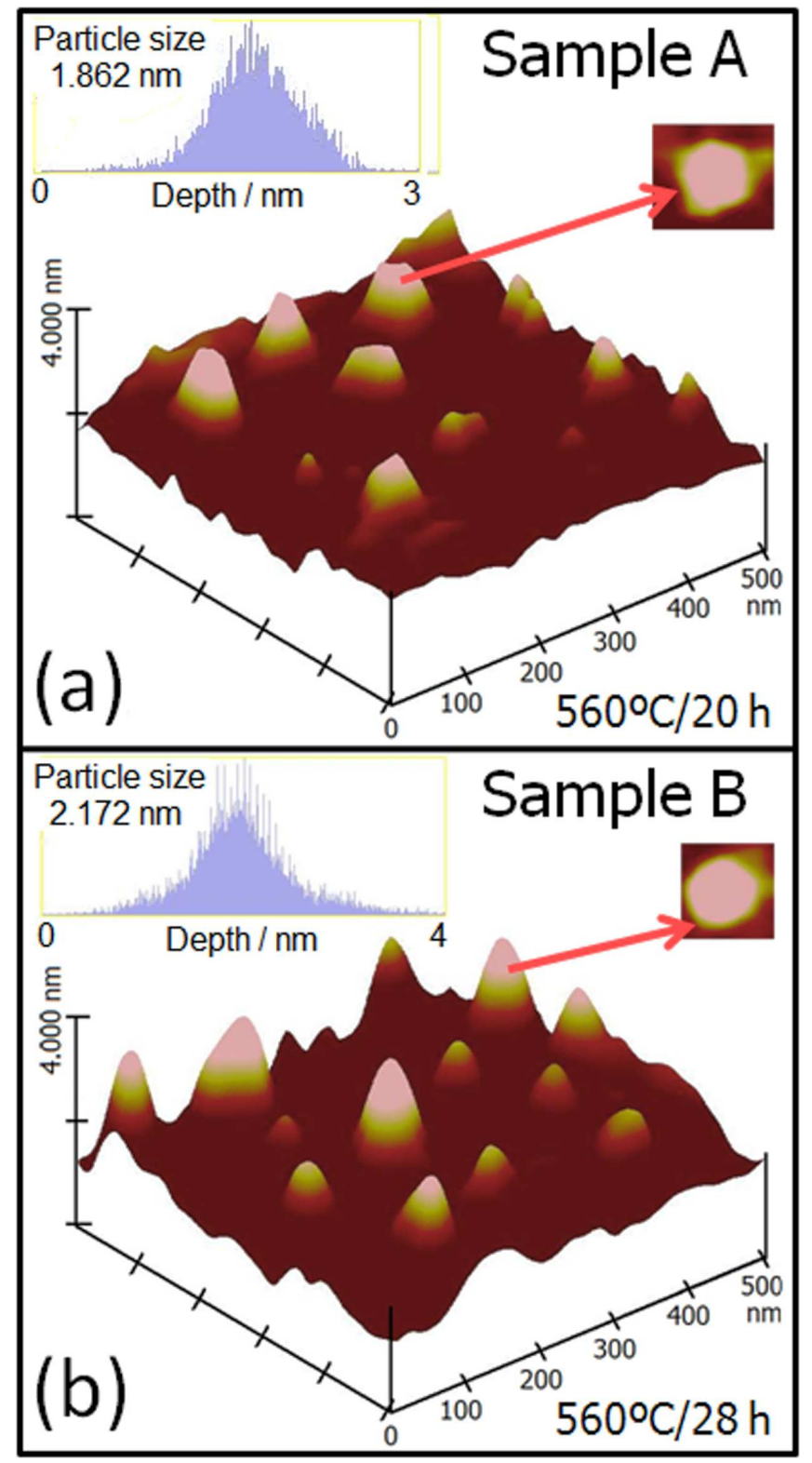

Figure 1. (colour online) AFM images and size distributions of CdSxSe1-x NCs embedded in a sample $A(a)$ and a sample $B(b)$, subjected to thermal annealing at $560^{\circ} \mathrm{C}$ for $20 \mathrm{~h}$ and $28 \mathrm{~h}$, respectively. A $2 \mathrm{D}$ image of isolated NCs is shown in detail. $80 \times 147 \mathrm{~mm}(400 \times 400 \mathrm{DPI})$ 

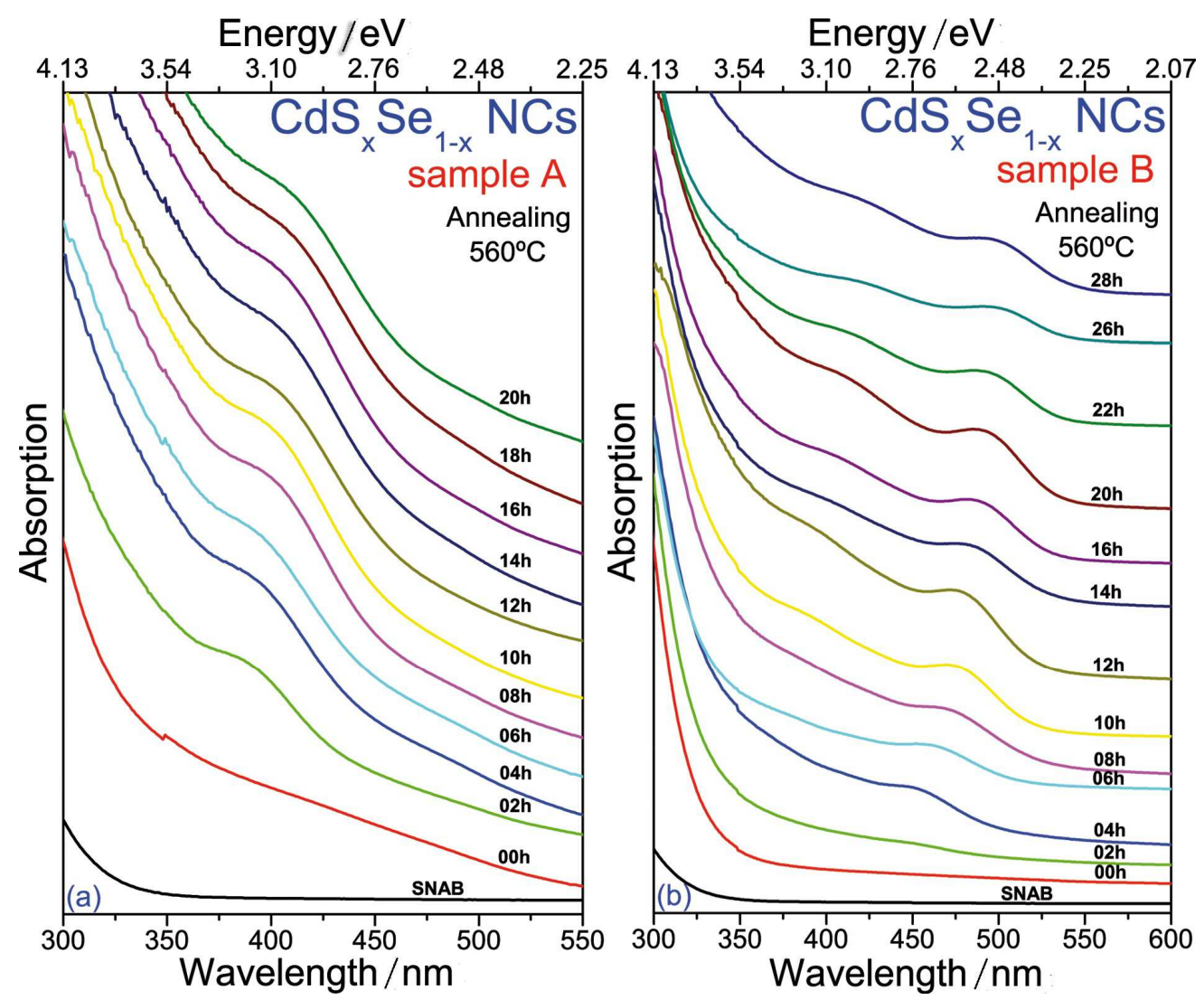

Figure 2. (colour online) Room temperature absorption spectra of samples A (a) and B (b) subjected to thermal annealing at $560^{\circ} \mathrm{C}$ for several hours. For comparison, it is also shown the absorption spectrum of SNAB glass matrix (bottom). $80 \times 66 \mathrm{~mm}(600 \times 600 \mathrm{DPI})$ 

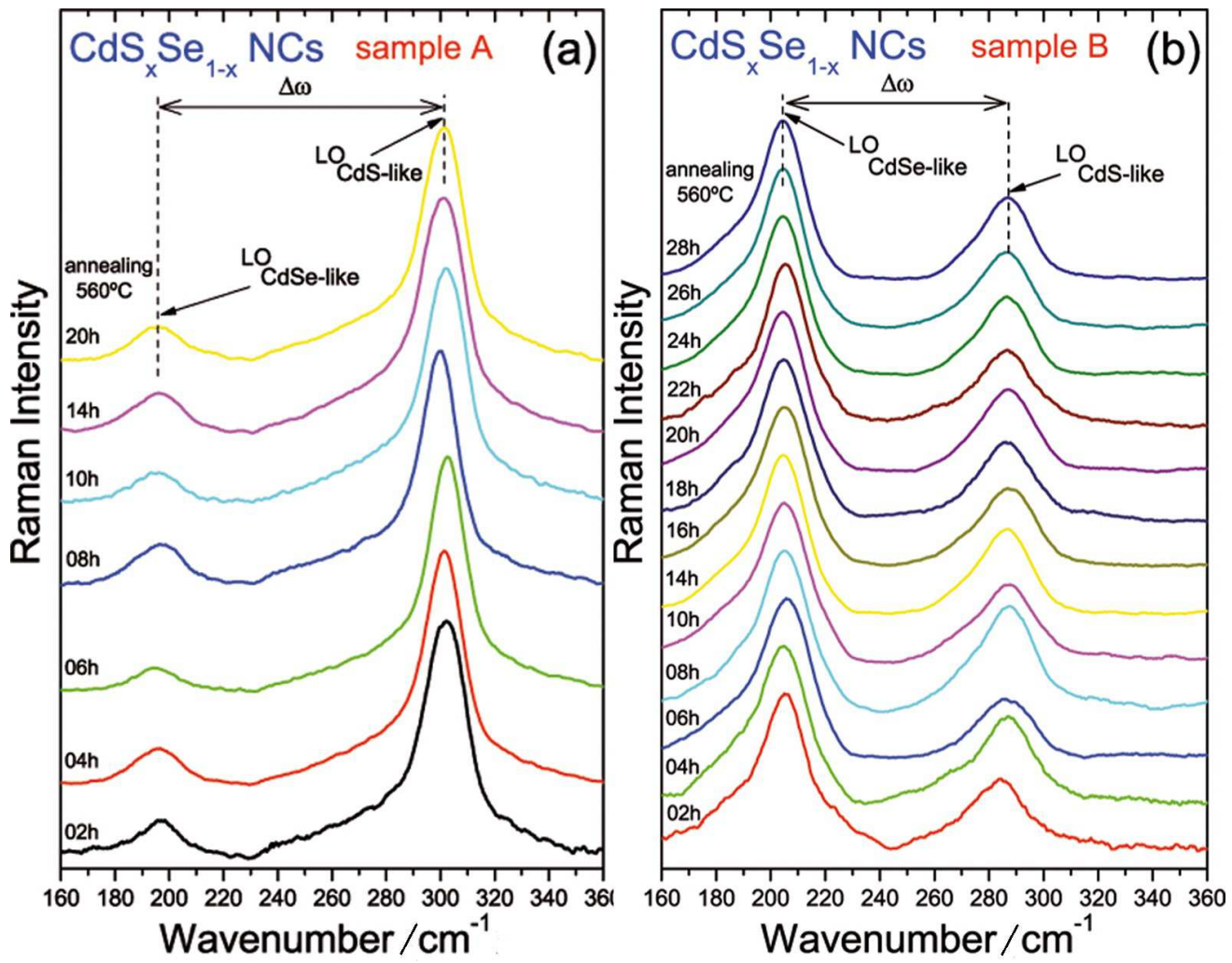

Figure 3. (colour online) Unpolarized RRS spectra recorded at room temperature from samples A (a) and samples $B(b)$ subjected to thermal annealing at $560^{\circ} \mathrm{C}$ for different time as indicated. $80 \times 62 \mathrm{~mm}(500 \times 500 \mathrm{DPI})$ 

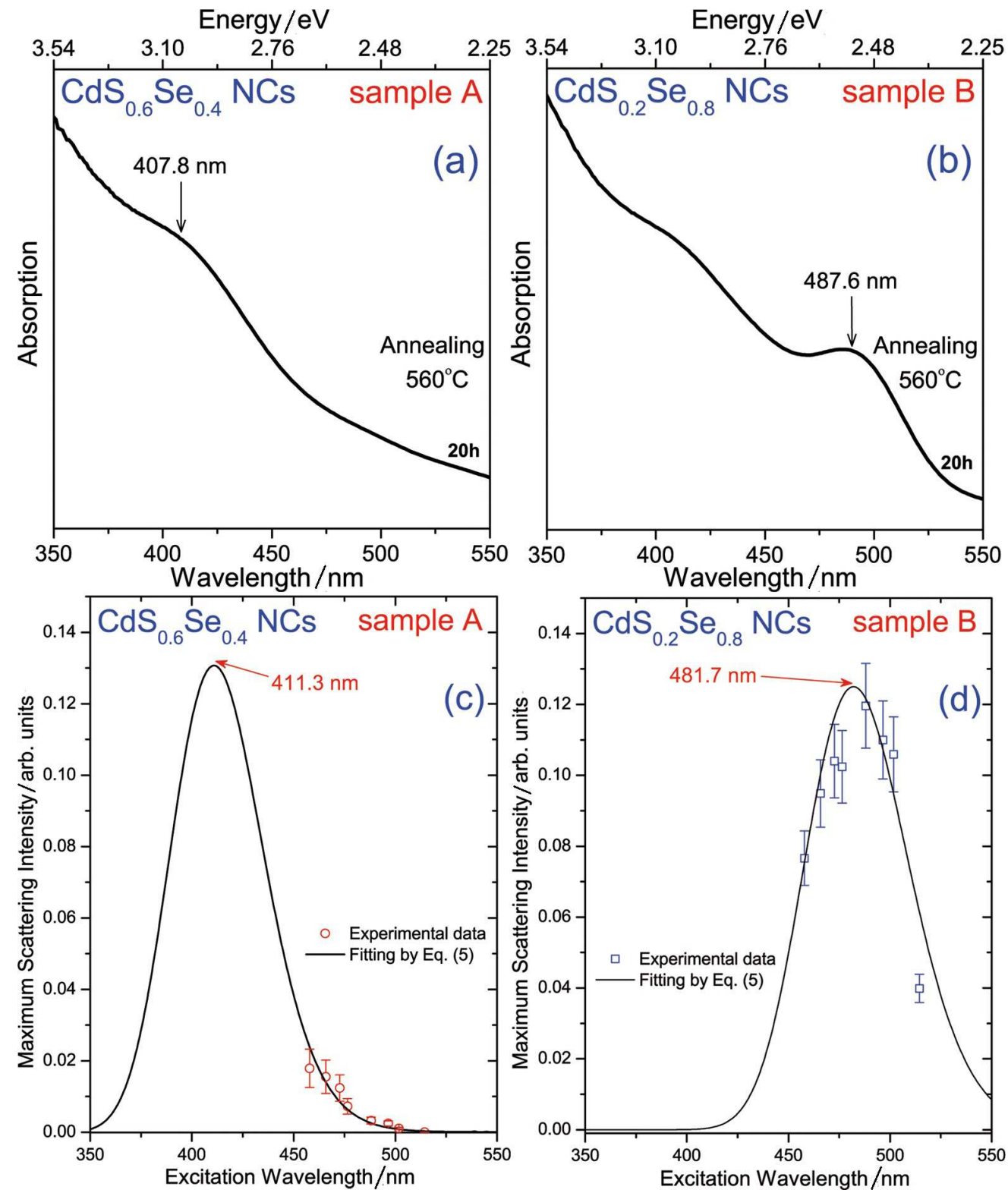

Figure 4. (colour online) Absorption spectra of the samples (a) A and (b) B subjected to thermal annealing for 20h. Resonant Raman curve of the corresponding samples (c) A and (d) B obtained from several excitation wavelengths. $80 \times 95 \mathrm{~mm}(500 \times 500 \mathrm{DPI})$ 

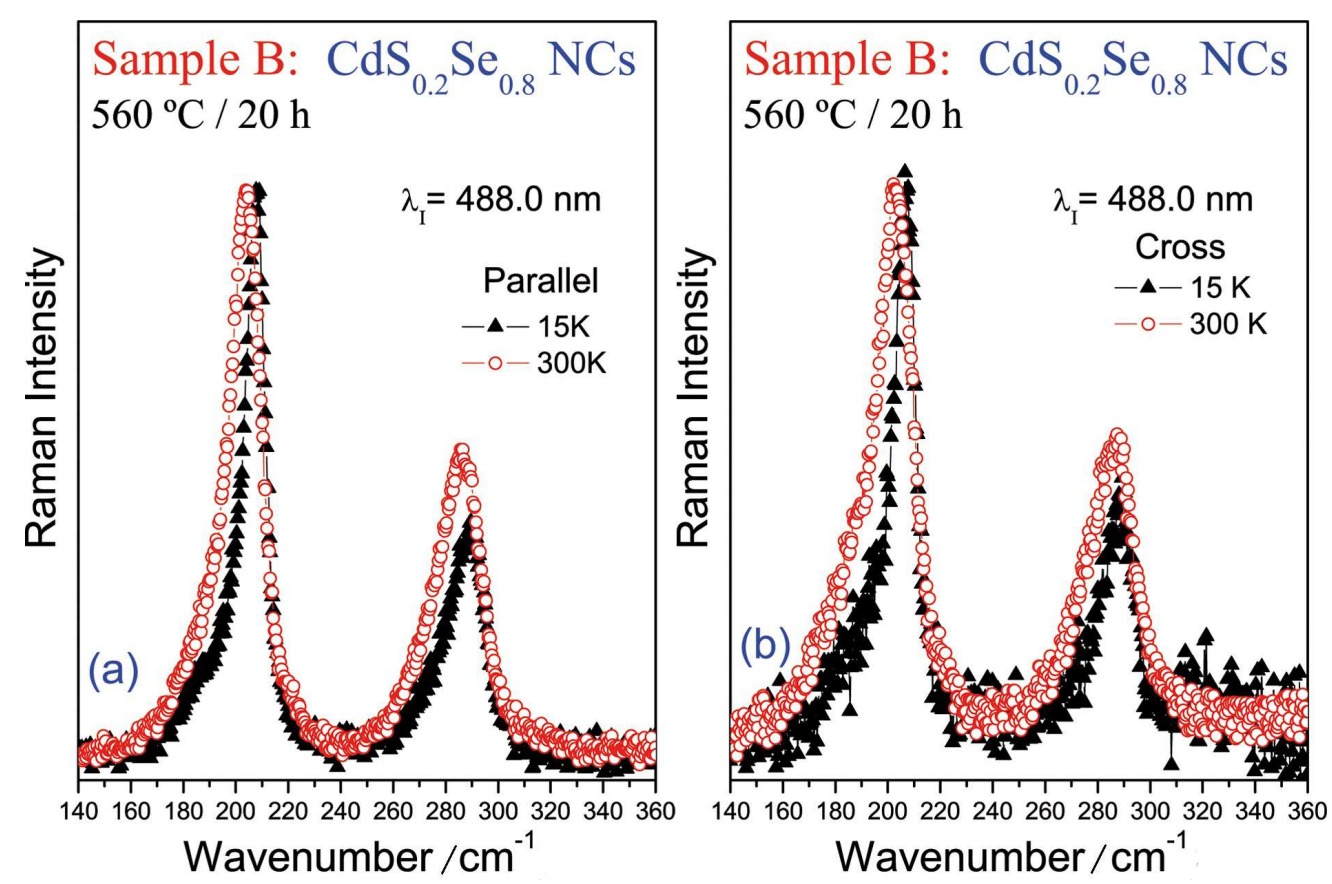

Figure 5. (colour online) Experimental RRS spectra ( $=488 \mathrm{~nm}$ ) with (a) parallel and (b) cross polarisations recorded at two different temperatures from the sample $B$ subjected to thermal annealing for $20 \mathrm{~h}$. $80 \times 52 \mathrm{~mm}(600 \times 600 \mathrm{DPI})$ 


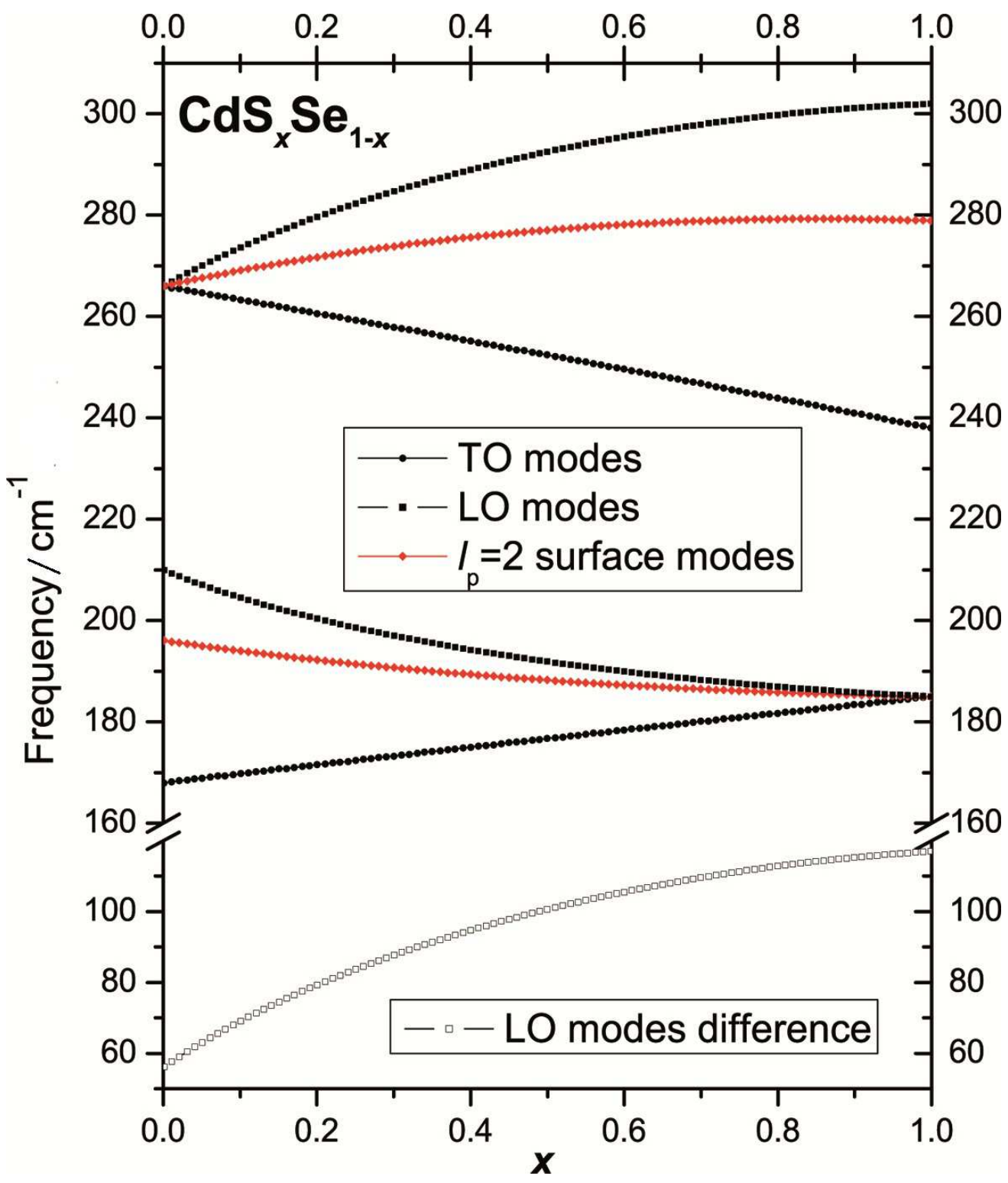

Figure 6. (colour online) Calculated frequencies of bulk optical phonons and electrostatic surface modes for CdSXSe1-x alloys of different composition. Also shown is the difference between the frequencies of the CdS-like and CdSe-like LO modes. The surface mode frequencies were calculated assuming for the surrounding medium. $59 \times 70 \mathrm{~mm}(500 \times 500 \mathrm{DPI})$ 

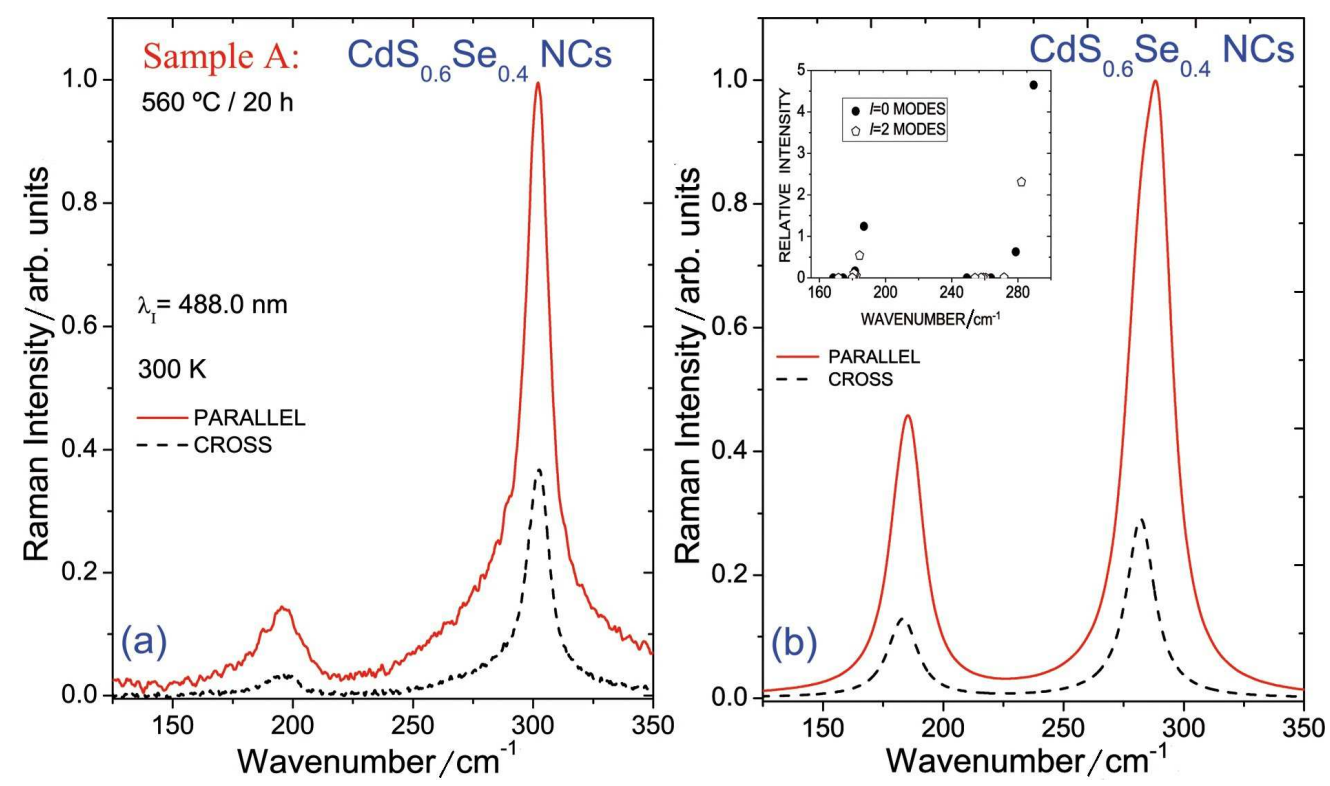

Figure 7. (colour online) Experimental ( $=488 \mathrm{~nm}$, panel a) and calculated (b) RRS spectra of sample A (annealed for $20 \mathrm{~h}$ ) for two polarizations of the scattered light. The inset shows relative contributions of the different confined phonon modes with angular momenta 0 and 2 to the parallel polarisation spectrum.

$80 \times 46 \mathrm{~mm}(600 \times 600 \mathrm{DPI})$ 

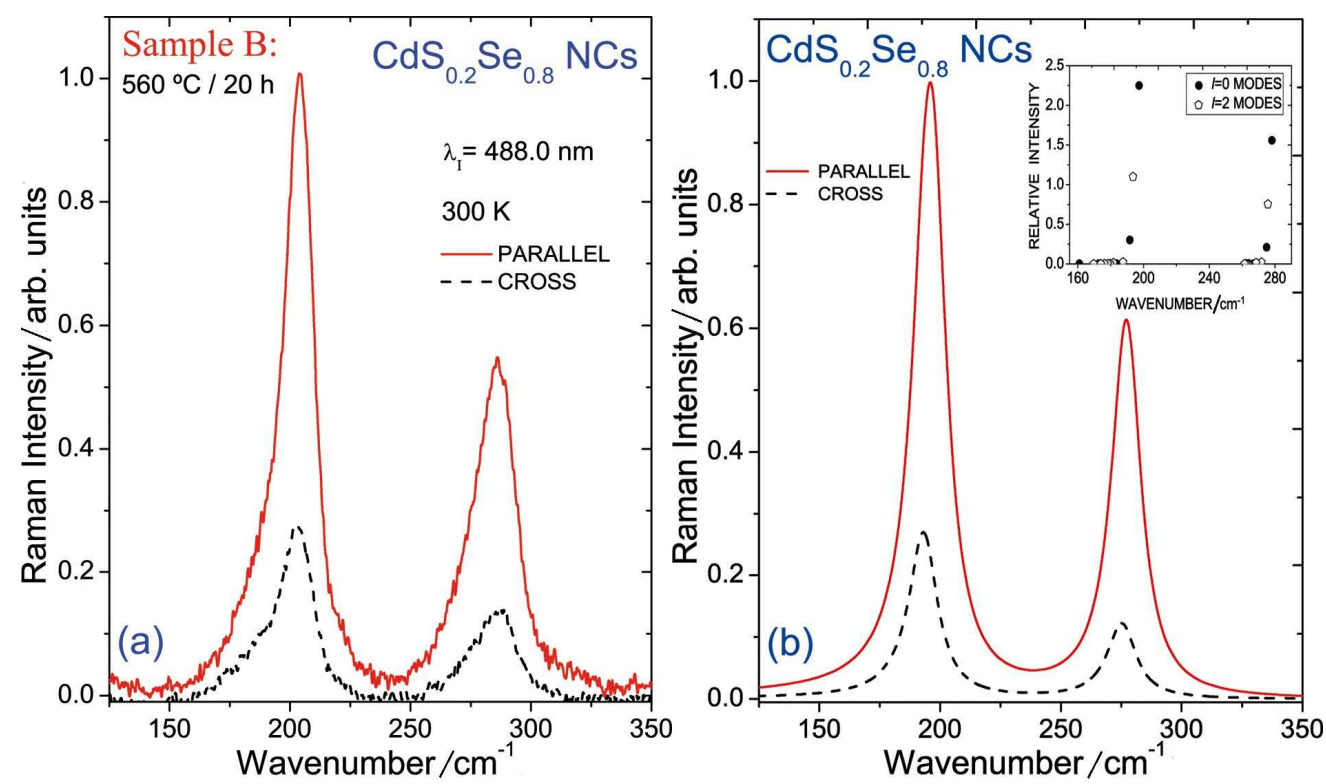

Figure 8. (colour online) Same as in Fig. 7 for sample B annealed for $20 \mathrm{~h}$. $80 \times 47 \mathrm{~mm}(600 \times 600$ DPI $)$ 